Dig Surg 1994;11:467-468

\title{
Subject Index Vol. 11, 1994
}

Abscess 209

Acute cholecystitis 6

disease 185

necrotizing pancreatitis 257

pancreatitis $192,213,225,230,244$

respiratory distress syndrome 230 Adenocarcinoma 25

-, gastric antrum 58 Adjuvant chemotherapy 111

therapy 408

treatment 377 Advanced gastric cancer 93,118 Amphiregulin 146 Amyloidosis 42

Anal canal 16

- $\quad$ manometry 20

Anastomotic insufficiency 78

Angiodysplasia 42

Angiography 350

Antibiotic(s) 213

-, prophylactic 219

AP-1 159

Ascites, pancreatic 451

Basic fibroblast growth factor 133 Biliary obstruction 365

- pancreatitis 225

Biopsy 350

Breast carcinoma, human 149 Brush cytology 345

Cancer 387

-, gallbladder 30

Carcinoid 456

Carcinoma 341

Caudate lobe 39

Celiac axis infusion 414

Cell lineage, ulcer-associated 142

Chemotherapy 118,408

-, adjuvant, intra-arterial 414

-, palliative, intra-arterial 414

-, pancreatic cancer 414

-, regional 414

Chronic inflammation 260

pancreatitis $137,192,274,290,299,303,312,330,397$

-, complications 303

-, etiology and outcome 266 
- $\quad$-, progressive/non-progressive 266

Cisplatin 118,414

Classification 285

Closed continuous lavage 244

Coffee 274

Colonic haemorrhage 42 Colorectal anastomosis 10 Common bile duct stones 225

Complications, adjacent organs 330 -, prevention 445 Computed tomography 350, 359

Cytokines 156

Delayed gastric emptying 397 Diabetes 182 Diagnosis 285,461 Diarrhea 438 Distal resection

312 Doxorubicin 118 Dumping syndrome 438 Duodenopancreatectomy 390 Duodenum-

preserving pancreatic head resection 317

- $\quad$ resection, head of pancreas 330

Early gastric cancer 64 Endocrine dysfunction 312

- $\quad$ secretion 433

Endometrial carcinoma, human 149 Endoscopic retrograde cholangiography 345

- cholangiopancreatography 365

sphincterotomy 219

therapy 290,299

ultrasonography 345 Epidermal growth factor 133, 142, 274

- - receptor 146, 156 Epirubicin 118

ERCP 225, 285

Esophageal variceal bleeding 438

Esophagectomy 1

Esophagus 25

Etoposide 118

Exocrine dysfunction 312

- $\quad$ secretion 433

Extended lymph node dissection 78

Faecal incontinence 20 Fistula in ano 16 Fistulas, enterocutaneous 451 -, pancreatic 451 18F-

Fluorodeoxyglucose 359 5-Fluorouracil 118 Folinicacid 414 5-Fu 414

Gallstone(s) 225

disease 185

pancreatitis 241

Gastrectomy, extent 58 Gastric cancer 64, 72, 78, 86, 93, 99, 104, 118,121

-, review 51

outlet obstruction 365

ulcer 27

Gastroenteropancreatic tumors 177, 461 Gene changes 163

- $\quad$ therapy 169

Giant tumor 30

Gliomas 149

Glucose transporters 359 Growth factors 137

Hartmann's procedure 10 Heparin-binding EGF 146 Hepatectomy 39 Hepatocellular carcinoma

39 Histopathology, early and late stages 266

Immunohistochemistry 137,149 Immunotherapy 156 Indomethacin 133 Infected pancreatic

necrosis 213,244 Infection route 213 In situ, hybridization 149 Insulin-like amyloid polypeptide 
182 Intensive care unit 230 Intraoperative radiation therapy 104 Ischemic injury 397 Islet transplantation 428 Isolation 428

K-ras oncogene 163

Laparoscopic surgery 64

Laparoscopy 10

Left diagonal thoracolaparotomy 1

- $\quad$ upper abdominal evisceration 78

Lesion-lifting method 64

Local resection 324 Lymphadenectomy 72, 86 Lymph node dissection 93

- $\quad$ - metastases 72

-, extent 104

Lysosomal enzymes 185

Malabsorption 299

Metal stent/endoprosthesis 365

Mitogen-activated protein kinase 159

- $\quad$ - - kinase 159

MitomycinC 118

Mitoxantrone 414

467

Morbidity 72 -, postoperative 402 Mortality, postoperative 402 Motility 433

Multimodality therapy 408 Multiple organ failure 230

Natural history 299 Necrosectomy 244,251 Necrosis 209

infected 219

- pancreatic 241 -, sterile 219

Necrotizing pancreatitis 230,244,251

Neoplasm(s) 350,359

NF-IL6 159

NK-кB 159

Noncompliance 86

Northern blot 359

Octreotide 433,438,445,451,456,461

Oncogene 169

Oncologic radicality 390

Open packing programmed lavage 257

Operative mortality 51

Over-80 age-group 6

Pain 260,299, 330 -, intractable 303

mechanisms 266

relief 312 Palliative treatment 121

Pancreas 341, 350, 359, 387,420,456

cancer 182

drainage 303

resection 303

Pancreatic cancer 156,159,163,169, 345, 365,371,377,390,408,414

-, human 146

carcinoma 274 
-, human 149

-, nonendocrine 337

duct 260

- obstruction 365

enzymes 299

fistula 78

neoplasm 36

secretion, exocrine 445

surgery 445

Pancreaticojejunostomy 312,324 Pancreatitis 185,209, 324, 359 -, acute 445 -, chronic 285, 317

Papillary-cystic neoplasm 36 Paraesophageal hernia 25 Pathology 337 Periampullary cancer 402

- neoplasm 397

Perineal descent 20

Perineurium 260

Peritoneal dissemination 93

Pharmacodynamics 433

Pharmacokinetics 433

Phytobezoar 27

Plastic stent/endoprosthesis 365

Polypoid cancer 30

Positron emission tomography

359 Postoperative complications 78 Prodrug activation 169 Prognosis 341 Progression 149

Prospective study 317 Pseudocyst 209 -, pancreatic 451 -, postnecrotic 266 Purification 428

Pylorus preservation 390 Pylorus-preserving pancreaticoduodenec-

tomy 312,397

- $\quad$ Whipple procedure 317

Quality control 86

Radionuclide studies 359

Radiotherapy 408

Receptors 137

Rectal neck 16

Rectum 16

Recurrence 99

Renal failure 42

Resection 341

-, extent 104

Respiration 1

Respiratory movement 1

Right posterolateral thoracotomy 1

RNA analysis 137

Scintigraphy 456 Sepsis 230 Serosal invasion 93 Short bowel syndrome 438

- $\quad$ hepatic vein 39

Side effects 461

Smoking 274

Somatostatin 433,438,445,451,456 
- $\quad$ receptor(s) 133,177

Spanchnic hemodynamics 433

Stage migration 86

Staging 285, 350 Stenting 290

Stomach neoplasm(s) 68, 111 Surgery 68, 99, 244, 387 -, indications 371 -, results 104,111

Surgical treatment 377 Survival 402 Survival, 5-year 51,58

Technology 350

Terlipressin 438

Trachea, membranous part 1

Transcription factors 159

Transforming growth factor- $\alpha 274$

- $\quad$ - factor- $31,-2$ and-3 149

Transplantation 420

Treatment 213,290,461

Trefoil peptides 142

Trichobezoar 27

Tubular complex 260 Tumor 456

- necrosis factor 156

Ulcer healing, experimental 133 Ultrasonography 209

Vasopressin 438 Videoproctogram 20

Wedge resection 64

468

Subject Index 